\title{
The isolation and molecular characterization of Leishmania spp. from patients with American tegumentary leishmaniasis in northwest Argentina
}

Department of Parasitology, Kochi Medical School, Kochi University, Nankoku, Kochi, Japan;

\section{Introduction}

Leishmaniasis is a zoonotic disease caused by kinetoplastid flagellates of the genus Leishmania. This tropical disease extends over 88 countries and it is considered as one of the most important diseases by the World Health Organization (WHO). In the Americas, leishmaniasis presents a variety of clinical manifestations which includes: visceral leishmaniasis (VL), the most severe form of the disease; mucocutaneous leishmaniasis (MCL), a mutilating disease; diffuse cutaneous leishmaniasis (DCL), a longlasting disease, and cutaneous leishmaniasis (CL), with a wide geographical distribution from the southern United States to the northern Argentina.

In Argentina, the primary focus of CL and MCL is found in the north of Salta Province. In this region, Leishmania (Viannia) braziliensis is the main causative agent, but L. (V.) guyanensis, L. (Leishmania) amazonensis and recently $L .(V$.$) panamensis and L .(L$.$) infantum have also been reported.$ An accurate method for identification of the different species is crucial for an effective treatment. Parasitological methods remain as the gold standard in leishmaniasis diagnosis because of its high specificity; however, these parasitological methods cannot differentiate parasite strains and/or species. Moreover, Multilocus Enzyme Electrophoresis (MLEE) is the gold standard for Leishmania spp.. Currently, PCR followed by sequencing of kinetoplastid cytochrome $b$ (cyt b) represent a alternative, being a validated method for the identification of the Leishmania spp.. In Argentina, Cyt b gene sequences have assigned two different genotypes for L. (V.) braziliensis named Ab-1 and Ab-2, in correspondence with the diversity detected by MLEE.

\section{Materials and methods}


Sixty six patients diagnosed with ATL, between July 2009 and November 2012, from Salta province, were included in this study. Two specimens were taken on glass slides from each patient's lesion stained by May Grünwald-Giemsa method. Each slide was observed in a search for Leishmania amastigotes using an optic microscope (1000X) for 40min. per 100 fields. Leishmanin skin test (LST) antigen derived from $L .(V$.$) braziliensis promastigotes parasite strain MHOM/AR/03/OLO1 was used for$ diagnosis in the study. After a positive ATL diagnosis, the patients were treated with $10-20 \mathrm{mg} / \mathrm{kg} /$ body weight of pentavalent antimony in a cycle of 25-30 days.

Lesion aspirate were inoculated in the "Difco" Blood agar (USMARU) medium, using PBSS+PE solution as liquid phase, and incubated at $24^{\circ} \mathrm{C}$. The WHO reference strains included in this work were: $L$. (V.) braziliensis: MHOM/BR/75/M2904; L. (V.) panamensis: MHOM/PA/71/LS94; L. (V.) guyanensis: MHOM/BR/75/M4147; L. (L.) amazonensis: MHOM/BR/73/M2269; L. (L.) mexicana: MNYC/BZ/62/M379; L. (L.) chagasi: MHOM/BR/74/PP75; and L. (L.) infantum: MHOM/TN/80/IPT1. Cultures in exponential growth were transferred to a culture tube with RPMI 1640 medium and cultured for 4 days, $100 \mu \mathrm{L}$ of the culture were sown in filter papers "Classic FTA Cards" (Whatman BioScience MA), in order to preserve and transport the material.

Classic FTA Cards filter paper fragments were processed according to manufacturer's instructions and used as a template for a nested PCR. The first reaction was performed with the degenerate primers for cyt b gene, L.cyt-AS (5'-GCGGAGAGRARGAAAAGGC-3') and L.cyt-AR(5'CCACTCATAAATATACTATA-3 ${ }^{\prime}$ ); and the primers L.cyt-S(5'-GGTGTAGGTTTTAGTYTAGG-3'); and L.cyt-R (5'-CTACAATAAACAAATCATAATATRCAATT-3') for the second reaction. We carried out double-stranded sequencing using BigDye terminator cycle sequencing kit, version 1.1 (Applied Biosystems, Foster City, CA), cleaned by Sephadex G-50 spin columns (Amersham Biosciences, NJ) with the primers L.cyt-S and L.cyt-R. Sequences were analyzed on an automated sequencer Applied Biosystems Hitachi 3130 Genetic Analyzer. The sequences were assembled and edited by Genetyx Mac 11.0.0 (Software Development Co. Ltd, Japan) and data were analyzed using the MLSTest v1.0 software. 
Stadistical analysis was performed with GraphPad Prism version 4 (GraphPad Software Inc., San Diego, USA), using data obtained from the patients interview of the diagnosed patients and the genotypes.

\section{Results}

In this study, culture technique reached a yield of Leishmania spp. isolation of $86.8 \%$ over 76 ATL cases caused by the Viannia subgenus parasites. Nested PCR and sequencing cyt b gene successfully identified two genotypes of $L$. (V.) braziliensis, 24 cases Ab-1 cyt b, 41 cases Ab-2 cyt b $(99.9 \%$ and 99.8\% similarity to the $L$. (V.) braziliensis MHOM/EC/88/INH-03 strain respectively), and one $L$. (V.) guyanensis strain (L. (V.) guyanensis reference strain MHOM/BR/75/M4147) obtained from a traveler from the Brazilian Amazon. Most of the cases typified in this study were registered in the area of Zenta Valley (Orán, Hipólito Yrigoyen, and Pichanal cities), being the only area in which the Ab-2 genotype was found, indicating a link between genotype and geographical origin. $92.4 \%$ of the cases were diagnosed as CL, while four 7.6\% corresponded to MCL form. The disease is distributed across all age groups and genders, although not uniformly, male in age groups between 16 to 60 years old were much more vulnerable to the disease. Forty-nine (78.1\%) of the patients presented one lesion; thirteen (14.6\%), two lesions; three (2.9\%) showed three lesions and one patient (1.6\%), four lesions on the body.

\section{Discussion}

In conclusion, the $c y t \mathrm{~b}$ sequencing method performed on templates from in vitro cultures improved the outcome of the identification of Leishmania spp. in northwest Argentina, allowing the largest isolation and typing of ATL cases in the northwest region. The use of in vitro cultures permits adjustments of the parasite DNA concentration, increasing the efficacy of PCR-based techniques.

Cyt $\mathrm{b}$ sequencing technique applied over the DNA material from promastigote cultures on FTA cards resulted in the incrimination of $L$. $(V$.$) braziliensis as the predominant species, apart from Ab-1 and$ Ab-2, no new genotypes were found. In addition, a correlation was found between genotype Ab-2 and the Zenta Valley area; indicating an unequal pattern in the distribution between Ab-1 and Ab-2, suggesting 
an association between the genotypes and different transmission cycles. It is worth noting that an isolate from a patient infected in Jujuy was assigned to $L$. (V.) braziliensis reported and isolated for the first time in this northwestern Argentinean province; and a strain identified as $L$. (V.) guyanensis isolated from a North American tourist infected while he was traveling through the Brazilian Amazon.

The transmission was predominantly associated with rural areas or rural activities, most of the patients being working age men, associated with rural areas in this study region. The male: female ratio (5:1) was higher than any other previously reported for Leishmaniasis in the Americas, and much higher compared with the disease ratio found in an urban area.

Cyt b sequencing allowed a precise identification at species level, but didn't provide much information about strain diversity. This fact highlights the necessity to explore new systems in order to provide a better understanding and knowledge of the transmission and the genetic diversity of parasites in this area. 